\title{
ON MODIFIED BOREL METHODS
}

\section{DIETER GAIER}

1. Introduction. Given a series $\sum a_{n}$ with partial sums $s_{n}$ it is possible to associate with it the Borel transforms

$$
B\left(x ; s_{k}\right)=e^{-x} \sum \frac{s_{k} x^{k}}{k !}, \quad B^{\prime}\left(x ; s_{k}\right)=\int_{0}^{x} e^{-t} a(t) d t, \quad a(t)=\sum \frac{a_{k} t^{k}}{k !}
$$

for $x>0$. One says that $B-\lim s_{n}=s\left[B^{\prime}-\lim s_{n}=s\right]$ if $\lim _{x \rightarrow \infty} B\left(x ; s_{k}\right)$ $=s\left[\lim _{x \rightarrow \infty} B^{\prime}\left(x ; s_{k}\right)=s\right]$. The relations between these Borel methods $B, B^{\prime}$, and their behavior under change of index are known [8, p. 183; $6 ; 7]$.

Following a suggestion of R. P. Boas, Jr., we intend to study in this paper the modified Borel methods which arise when the continuous variable $x$ in (1.1) is replaced by the discrete sequence of integers $n=1,2, \cdots$. The resulting methods shall be denoted by $B_{I}$ and $B_{I}^{\prime}$, and our interest is to discuss the relations among the methods $B$, $B_{I}, B^{\prime}, B_{I}^{\prime}$ (which is done in $\S 3$ ) and the behavior of these methods under change of index (cf. §4). The methods $B_{I}, B_{I}^{\prime}$ show certain abnormalities in comparison with $B, B^{\prime}$. For example, $B-\lim s_{n}=s$ always implies $B^{\prime}-\lim s_{n}=s$, whereas $B_{I}-\lim s_{n}=s$ implies $B_{I}^{\prime}-\lim s_{n}=s$ if $a_{n}=O\left(K^{n}\right)$ for $K<\left(\pi^{2}+1\right)^{1 / 2}$ and not for $K=\left(\pi^{2}+1\right)^{1 / 2}$.

Our results are based on two theorems on entire functions ( $\$ 2)$. The first allows one to infer $f(x) \rightarrow s[x \rightarrow+\infty]$ from $f(n) \rightarrow s(n=1,2, \cdots)$ if the type of $f(z)$ is less than $\pi$, and is well known; the second allows one to infer $f(x) \cong s e^{x}[x \rightarrow+\infty]$ from $f(n) \cong s e^{n}(n=1,2, \cdots)$ if the type of $f(z)$ is less than $\left(\pi^{2}+1\right)^{1 / 2}$.

Finally, in $\$ 5$ Cesàro-Borel methods are considered but the results there are incomplete, whereas the results in $\$ 3$ and $\$ 4$ are in a certain sense best possible.

2. A theorem on functions of exponential type. If $f(z)$ is regular in the angle $|\arg z| \leqq \alpha(\alpha>0)$, it is said to be there of exponential type $\tau$ if for every $\epsilon>0$, but for no $\epsilon<0$, there exists a constant $M(\epsilon)$ such that

$$
|f(z)| \leqq M(\epsilon) e^{(\tau+e)|z|} \quad(|\arg z| \leqq \alpha) .
$$

The growth of $f(z)$ along the ray arg $z=\phi(|\phi| \leqq \alpha)$ is described by the indicator function

Received by the editors January 14, 1955. 


$$
h_{f}(\phi)=\limsup _{r \rightarrow \infty} r^{-1} \log \left|f\left(r e^{i \phi}\right)\right| .
$$

In $\S 3$ and $\S 4$ we meet the problem of going from the behavior of $f(n)(n=1,2, \cdots)$ to the behavior of $f(x)(x \rightarrow+\infty)$. A well known theorem in this direction is ${ }^{1}$

THEOREM 1. If $f(z)$ is regular and of exponential type in $|\arg z|$ $\leqq \alpha \leqq \pi / 2(\alpha>0)$, and if

$$
h_{f}( \pm \alpha)<\pi \sin \alpha,
$$

then $f(n) \rightarrow 1(n=1,2, \cdots)$ implies $f(x) \rightarrow 1(x \rightarrow+\infty)$.

For our purposes we need an extension of Theorem 1 covering the case $f(n) \cong e^{n}$ instead of $f(n) \rightarrow 1$.

THEOREM 2. If $f(z)$ is regular and of exponential type in $|\arg z|$ $\leqq \alpha \leqq \pi / 2(\alpha>0)$, and if

$$
h_{f}( \pm \alpha)<\pi \sin \alpha+a \cos \alpha \quad(a \geqq 0),
$$

then

(2.2) $f(n) \cong n^{k} \cdot e^{a n}(n=1,2, \cdots)$ implies $f(x) \cong x^{k} \cdot e^{a x}(x \rightarrow+\infty)(k \geqq 0)$.

In particular, (2.2) is true if $f(z)$ is regular and of exponential type $\tau<\left(\pi^{2}+a^{2}\right)^{1 / 2}$ in $\Re(z) \geqq 0$; for $\tau=\left(\pi^{2}+a^{2}\right)^{1 / 2}$ this is false.

Proof. Consider $g(z)=f(z) e^{-a z}(z+1)^{-k}$ in $|\arg z| \leqq \alpha$, where $(z+1)^{k}$ is assumed to be $>0$ for $z>0$. For the indicator function of $g(z)$ on $\arg z= \pm \alpha$ we have

$$
\begin{aligned}
h_{o}( \pm \alpha) & =\limsup _{r \rightarrow \infty} r^{-1} \log \left|g\left(r e^{ \pm i \alpha}\right)\right| \\
& =\limsup _{r \rightarrow \infty} r^{-1} \log \left|f\left(r e^{ \pm i \alpha}\right)\right|-a \cos \alpha<\pi \sin \alpha
\end{aligned}
$$

by (2.1), and hence, by Theorem $1, g(n) \rightarrow 1(n=1,2, \cdots)$ implies $g(x) \rightarrow 1(x \rightarrow+\infty)$.

If, in particular, $f(z)$ is of exponential type $\tau<\left(\pi^{2}+a^{2}\right)^{1 / 2}$ in $\Re(z)$ $\geqq 0$, we choose $\alpha$ such that $\operatorname{tg} \alpha=\pi / a$, so that

$$
\pi \sin \alpha+a \cos \alpha=\pi / \sin \alpha=\left(\pi^{2}+a^{2}\right)^{1 / 2}>\tau \geqq h_{f}( \pm \alpha),
$$

i.e. hypothesis (2.1) is fulfilled and hence (2.2) follows.

For the last part of the theorem consider $f(z)=e^{a z}(\sin \pi z+1)$.

1 Theorem 1 is implicitly contained in Cartwright [2], explicitly in Macintyre $[9$, p. 16]. See also Pfluger [12, pp. 312-314], Duffin-Schaeffer [5, pp. 142-143] and Boas [1, p. 180]. 
3. Relations between the Borel methods. Now we are going to consider the methods of summability which associate with a given series the following transformations:

$B: e^{-x} \sum \frac{s_{k} x^{k}}{k !}(x>0)$;

$$
\begin{aligned}
B^{\prime}: \int_{0}^{x} e^{-t} a(t) d t(x>0) & \\
a(t) & =\sum \frac{a_{k} t^{k}}{k !},
\end{aligned}
$$

$B_{I}: e^{-n} \sum \frac{s_{k} n^{k}}{k !}(n=1,2, \cdots) ; B_{I}^{\prime}: \int_{0}^{n} e^{-t} a(t) d t(n=1,2, \cdots)$.

The $B$ - and $B^{\prime}$-transformations are connected by the formal relation (Hardy [8, p. 182])

$$
\begin{aligned}
B\left(x ; s_{k}\right) & =B\left(x ; a_{k}\right)+B^{\prime}\left(x ; s_{k}\right), \text { i.e. } \\
e^{-x} \sum \frac{s_{k} x^{k}}{k !} & =e^{-x} \sum \frac{a_{k} x^{k}}{k !}+\int_{0}^{x} e^{-t} a(t) d t .
\end{aligned}
$$

The problem of this paragraph is to investigate the relative strength of the above Borel methods. For two summability methods $V_{1}$ and $V_{2}$ we use the notation $V_{1} \rightarrow V_{2}$, if $V_{1}$-lim $s_{n}=s$ implies always $V_{2}-\lim s_{n}=s$.

The following relations are trivial or known.

(3.2) $B \rightarrow B_{I}$ and $B^{\prime} \rightarrow B_{I}^{\prime}$.

(3.3) $B \rightarrow B^{\prime}$ (Hardy [8, p. 183]).

(3.4) $B^{\prime} \rightarrow B$ if $a_{n}=O\left(K^{n}\right)$ for some $K>0$ (Gaier [6, p. 455]). This becomes false if $a_{n}=O\left(K^{n}\right)$ is replaced by $a_{n}=O\left(n^{\epsilon n} K^{n}\right)$ ( $\epsilon$ arbitrary $>0$ ) (Gaier [7]).

Our new results about the relations between the Borel methods are summarized in

Theorem 3. (1) $B_{I} \rightarrow B, B_{I} \rightarrow B^{\prime}$, and $B_{I} \rightarrow B_{I}^{\prime}$, if $a_{n}=O\left(K^{n}\right)$ for $K<\left(\pi^{2}+1\right)^{1 / 2}$, but not for $K=\left(\pi^{2}+1\right)^{1 / 2}$.

(2) $B_{I}^{\prime} \rightarrow B^{\prime}, B_{I}^{\prime} \rightarrow B$, and $B_{I}^{\prime} \rightarrow B_{I}$, if $a_{n}=O\left(K^{n}\right)$ for $K<\left(\pi^{2}+1\right)^{1 / 2}$, but not for $K=\left(\pi^{2}+1\right)^{1 / 2}$.

Note, in particular, that there is no analogy to (3.3) for the methods $B_{I}$ and $B_{I}^{\prime}$.

Proof. (1) (a) $B_{I} \rightarrow B$. (i) If $a_{n}=O\left(K^{n}\right)\left(K<\left(\pi^{2}+1\right)^{1 / 2}\right)$, then $\left|s_{n}\right| \leqq M K^{\prime n}\left(K^{\prime}<\left(\pi^{2}+1\right)^{1 / 2}\right)$ and the entire function $\phi(z)=\sum s_{n} z^{n} / n$ ! satisfies the estimation

$$
|\phi(z)| \leqq M \sum \frac{K^{\prime n}|z|^{n}}{n !}=M e^{K^{\prime}|z|}
$$


i.e. it is of type $\tau<\left(\pi^{2}+1\right)^{1 / 2}$. Therefore the assumption $\phi(n) \cong A \cdot e^{n}$ $(n=1,2, \cdots)$ implies, by Theorem $2, \phi(x) \cong A \cdot e^{x}(x \rightarrow+\infty)$, i.e. $B-\lim s_{n}=A$.

(ii) Define $s_{n}$ by $\sum\left(s_{n} z^{n} / n !\right)=e^{z}(\sin \pi z+1)$. Then $(\alpha) B_{I}-\lim s_{n}=1$, but not $B-\lim s_{n}=1$. ( $\left.\beta\right)$ One finds immediately

$$
s_{n}=1+(1 / 2 i)\left\{(1+i \pi)^{n}-(1-i \pi)^{n}\right\},
$$

so that $s_{n}=O\left(\left(\pi^{2}+1\right)^{n / 2}\right)$ and also $a_{n}=O\left(\left(\pi^{2}+1\right)^{n / 2}\right)$ are fulfilled.

(b) $B_{I} \rightarrow B^{\prime}$. (i) The assumption about the $a_{n}$ implies (Case (a) and (3.3))

$$
B_{I} \rightarrow B \rightarrow B^{\prime} .
$$

(ii) Define $s_{n}$ as above. Then $(\alpha) B_{I}-\lim s_{n}=1$, but not $B^{\prime}-\lim s_{n}=1$; otherwise $B^{\prime}-\lim s_{n}=1$ would by (3.4) imply $B$-lim $s_{n}=1$ which is false. $(\beta) a_{n}=O\left(\left(\pi^{2}+1\right)^{n / 2}\right)$ is fulfilled.

(c) $B_{I} \rightarrow B_{I}^{\prime}$. (i) The assumption about the $a_{n}$ implies (Case (b) and (3.2))

$$
B_{I} \rightarrow B^{\prime} \rightarrow B_{I}^{\prime}
$$

(ii) Define $a_{n}$ by

$$
\int_{0}^{z} e^{-t} a(t) d t=\sin (\pi z+\alpha) ; \quad \operatorname{tg} \alpha=-\pi .
$$

Then $(\alpha) B_{I}-\lim s_{n}=0$. For, by the relation (3.1), we have

$$
B\left(x ; s_{k}\right)=\frac{d}{d x} \sin (\pi x+\alpha)+\sin (\pi x+\alpha),
$$

which, taken at $x=n(n=1,2, \cdots)$, becomes

$$
B\left(n ; s_{k}\right)=\cos \pi n(\sin \alpha+\pi \cos \alpha)=0 \quad(n=1,2, \cdots) .
$$

On the other hand $B_{I}^{\prime}-\lim s_{n}$ does not exist. $(\beta)$ We have

$$
a(t)=e^{t} \cdot \pi \cos (\pi t+\alpha)=\sum \frac{a_{k} t^{k}}{k !}
$$

from which $a_{n}=O\left(\left(\pi^{2}+1\right)^{n / 2}\right)$ is immediate.

(2) (a) $B_{I}^{\prime} \rightarrow B^{\prime}$. (i) If $a_{n}=O\left(K^{n}\right)\left(K<\left(\pi^{2}+1\right)^{1 / 2}\right)$, then $a(t)$ is an entire function of exponential type $\tau<\left(\pi^{2}+1\right)^{1 / 2}$. If therefore $g(z)$ $=e^{-z} a(z)$, we have for the indicator function of $g(z)$ taken for the rays $\arg z= \pm \alpha(\operatorname{tg} \alpha=\pi)$

$$
h_{\theta}( \pm \alpha)=h_{a}( \pm \alpha)-\cos \alpha<\left(\pi^{2}+1\right)^{1 / 2}-\cos \alpha=\pi \sin \alpha,
$$


and hence for the function $\phi(z)=\int_{0}^{z} e^{-t} a(t) d t$

$$
h_{\phi}( \pm \alpha)<\pi \sin \alpha,
$$

so that an application of Theorem 1 infers $\phi(x) \rightarrow A(x \rightarrow+\infty)$ from $\phi(n) \rightarrow A(n=1,2, \cdots)$.

(ii) Define $a_{n}$ by $\int_{0}^{z} e^{-t} a(t) d t=\sin \pi z$. Obviously $B_{I}^{\prime}-\lim s_{n}=0$, but not $B^{\prime}-\lim s_{n}=0$. The validity of $a_{n}=O\left(\left(\pi^{2}+1\right)^{n / 2}\right)$ is again immediate.

(b) $B_{I}{ }^{\prime} \rightarrow B$. (i) The assumption about the $a_{n}$ implies (Case (a) and (3.4))

$$
B_{I}^{\prime} \rightarrow B^{\prime} \rightarrow B
$$

(ii) Define $a_{n}$ as in (2) (a). $B$-lim $s_{n}=0$ cannot hold since $B^{\prime}$-lim $s_{n}$ does not exist.

(c) $B_{I} \rightarrow B_{I}$. (i) By the preceding case $B_{I}^{\prime} \rightarrow B \rightarrow B_{I}$.

(ii) Define $a_{n}$ as in (2) (a). By (3.1), the $B$-transform of the corresponding sequence $s_{n}$ is $\sin \pi x+\pi \cos \pi x$, so that $B_{I}\left(n ; s_{k}\right)= \pm \pi$ $(n=1,2, \cdots)$.

4. On the change of index for the methods $B_{I}$ and $B_{I}^{\prime}$. We consider the two series

$$
\sum a_{k}=a_{0}+a_{1}+a_{2}+\cdots \quad \text { with partial sums } s_{n}
$$

and

$$
\sum b_{k}=0+a_{0}+a_{1}+\cdots \quad \text { with partial sums } t_{n} .
$$

The problem is to determine under what conditions
(4.1.a)
$V$ - $\lim s_{n}=s$ implies $V$ - $\lim t_{n}=s$

or

$$
V-\lim t_{n}=s \text { implies } V-\lim s_{n}=s,
$$

where $V$ is one of the methods $B_{I}, B_{I}^{\prime} .^{2}$

In addition to (3.1) we shall need the relations

$$
B^{\prime}\left(x ; s_{k}\right)=B\left(x ; t_{k}\right)
$$

and

$$
B^{\prime}\left(x ; s_{k}\right)=B\left(x ; b_{k}\right)+B^{\prime}\left(x ; t_{k}\right) .
$$

${ }^{2}$ This problem has been treated for other methods of summability; cf. Doetsch [4], p. 464 ff. for $B$; Doetsch [3], for $C_{k} B$; Gaier [6] and [7] for $B$; Meyer-König [10, p. 270] and Meyer-König and Zeller [11, pp. 348-349] for $T_{\alpha}, S_{\alpha}, T_{\alpha}^{\prime} ;$ Wollan [13, p. 583] for Euler summability of double series. 
Note that $B\left(x ; b_{k}\right)=(d / d x) B^{\prime}\left(x ; t_{k}\right)$. The proof of (4.3) follows from $^{8}$

$$
\begin{aligned}
B^{\prime}\left(x ; t_{k}\right)= & \int_{0}^{x} e^{-t} b(t) d t=-\left.e^{-t} b(t)\right|_{0} ^{x} \\
& +\int_{0}^{x} e^{-t} a(t) d t=-B\left(x ; b_{k}\right)+B^{\prime}\left(x ; s_{k}\right) .
\end{aligned}
$$

THEOREM 4. If $V$ is one of the methods $B_{I}, B_{I}^{\prime}$, both statements (4.1.a) and (4.1.b) are correct if $a_{n}=O\left(K^{n}\right)$ for $K<\left(\pi^{2}+1\right)^{1 / 2}$, but not for $K=\left(\pi^{2}+1\right)^{1 / 2}$.

Note, in particular, that there is no analogy to the fact that (4.1.a) holds for $V=B$ without restriction of the $a_{n}$.

Proof. (1) $V=B_{I}$. (a) By (4.2), $B_{I}-\lim t_{n}=s$ if and only if $B_{I}^{\prime}-\lim s_{n}$ $=s$, which follows from $B_{I}-\lim s_{n}=s$ if $a_{n}=O\left(K^{n}\right)$ for $K<\left(\pi^{2}+1\right)^{1 / 2}$, but not for $K=\left(\pi^{2}+1\right)^{1 / 2}$ (Theorem 3, 1c).

(b) Again, $B_{I}$-lim $t_{n}=s$ if and only if $B_{I}^{\prime}-\lim s_{n}=s$, which implies $B_{I}-\lim s_{n}=s$ if $a_{n}=O\left(K^{n}\right)$ for $K<\left(\pi^{2}+1\right)^{1 / 2}$, but not for $K=\left(\pi^{2}+1\right)^{1 / 2}$ (Theorem 3, 2c).

(2) $V=B_{I}^{\prime}$. (a) (i) If $a_{n}=O\left(K^{n}\right)\left(K<\left(\pi^{2}+1\right)^{1 / 2}\right), B_{I}^{\prime}-\lim s_{n}=s$ implies $B^{\prime}$-lim $s_{n}=s$ so that by $(4.3) \phi(x)+\phi^{\prime}(x) \rightarrow s(x \rightarrow+\infty)\left[\phi^{\prime}(x)\right.$ $\left.=B\left(x ; b_{k}\right)\right]$, and consequently (Hardy $[8$, p. 107]) $\phi(x) \rightarrow s(x \rightarrow+\infty)$, i.e. $B_{I}{ }^{\prime}-\lim t_{n}=s$.

(ii) Define $b_{n}$ by $\int_{0}^{x} e^{-t} b(t) d t=\sin (\pi x+\alpha)$ with $\operatorname{tg} \alpha=-\pi$ and proceed as in Theorem 3,1 (c) (ii). We get $B_{I}^{\prime}-\lim s_{n}=0$ whereas $B_{I}^{\prime}-\lim t_{n}$ does not exist, although $a_{n}=O\left(\left(\pi^{2}+1\right)^{n / 2}\right)$.

(b) (i) If $a_{n}=O\left(K^{n}\right)\left(K<\left(\pi^{2}+1\right)^{1 / 2}\right), B_{I}^{\prime}-\lim t_{n}=s$ implies $B^{\prime}-\lim t_{n}$ $=s$ (Theorem 2), and since $B^{\prime}\left(z ; t_{k}\right)$ is an entire function of exponential type tending to $s$ as $z \rightarrow+\infty$, its derivative $e^{-z} b(z)=B\left(z ; b_{k}\right)$ tends to zero as $z \rightarrow+\infty$ (Boas $[1$, p. 212] and Gaier [6, p. 454]) which, by (4.3), implies $B_{I}^{\prime}-\lim s_{n}=s$.

(ii) Define $b_{n}$ by $\int_{0}^{x} e^{-t} b(t) d t=\sin \pi x$. Then $B_{I}^{\prime}-\lim t_{n}=0$, but not $B_{I}^{\prime}-\lim s_{n}=0$, although $a_{n}=O\left(\left(\pi^{2}+1\right)^{n / 2}\right)$.

5. Cesàro-Borel methods. Doetsch [3] was the first to consider the Cesàro-Borel transform

$$
C_{k} B\left(x ; s_{k}\right)=k x^{-k} \int_{0}^{x} B\left(t ; s_{k}\right)(x-t)^{k-1} d t \quad(k>0, x \geqq 0),
$$

and in view of our results in $\S 3$ one can ask what relations there are

- Let $b(t)=\sum\left(b_{n} t^{n} / n !\right)$, so that $b^{\prime}(t)=a(t)$. 
for example between the methods $C_{k} B$ and $C_{k} B_{I}$ ( $C_{k}=$ matrix method in the latter case). It is not surprising that in general

$$
C_{k} B_{I}-\lim s_{n}=s \quad \text { does not imply } \quad C_{k} B \text { - } \lim s_{n}=s ;
$$

however, also

$$
C_{k} B-\lim s_{n}=s \text { does not imply } C_{k} B_{I}-\lim s_{n}=s .
$$

Equivalent to the problem raised is, of course, under what conditions for an entire function $f(z)$ does

$$
C_{k}-\lim f(n)=s \text { imply } C_{k}-\lim f(x)=s
$$

and conversely. For $k=1$ the statement (5.1) follows from consideration of $f(z)=z \sin \pi z$, whereas for the proof of (5.2) we take an entire function $f(z)$ of exponential type $(<2 \pi+\epsilon)$ which is, for $x>0$,

$$
f(x)=x^{1 / 2} \cos 2 \pi x+o(1) .^{4}
$$

Then obviously $C_{1}-\lim f(n)=+\infty$, but $C_{1}-\lim f(x)=0$. The author has no contribution towards the solution of this problem.

\section{REFERENCES}

1. R. P. Boas, Jr., Entire functions, New York, 1954.

2. M. L. Cartwright, On certain integral functions of order 1, Quart. J. Math. Oxford Ser. vol. 7 (1936) pp. 46-55.

3. G. Doetsch, Eine neue Verallgemeinerung der Borelschen Summabilitätstheorie der divergenten Reihen, Dissertation, Göttingen, 1920.

4. - Handbuch der Laplace-Transformation I, Basel, 1950.

5. R. J. Duffin and A. C. Schaeffer, Power series with bounded coefficients, Amer. J. Math. vol. 67 (1945) pp. 141-154.

6. D. Gaier, Zur Frage der Indexverschiebung beim Borel-Verfahren, Math. Zeit. vol. 58 (1953) pp. 453-455.

7. - On the change of index for summable series, to appear in Pacific Journal of Mathematics.

8. G. H. Hardy, Divergent series, Oxford, 1949.

9. A. J. Macintyre, Laplace's transformation and integral functions, Proc. London Math. Soc. (2) vol. 45 (1938-1939) pp. 1-20.

10. W. Meyer-König, Untersuchungen über einige verwandte Limitierungsverfahren, Math. Zeit. vol. 52 (1949) pp. 257-304.

11. W. Meyer-König and K. Zeller, Über das Taylorsche Summierungsverfahren, Math. Zeit. vol. 60 (1954) pp. 348-352.

12. A. Pfluger, On analytic functions bounded at the lattice points, Proc. London Math. Soc. (2) vol. 42 (1936-1937) pp. 305-315.

13. G. N. Wollan, On Euler methods of summability for double series, Proc. Amer. Math. Soc. vol. 4 (1953) pp. 583-587.

\section{Technische Hochschule, Stuttgart}

'To obtain such a function apply Macintyre's lemma $\left[1\right.$, p. 80] to $f(z)=z^{1 / 2}$ $\cdot \cos 2 \pi z ; f(z / 2+\epsilon)$ is of type $<\pi$ in $\Re(z) \geqq 0$. 